\title{
Impact on Spin Tune From Horizontal Orbital Angle Between Snakes and Orbital Angle Between Spin Rotators
}

\author{
M. Bai, V. Ptitsyn, T. Roser
}

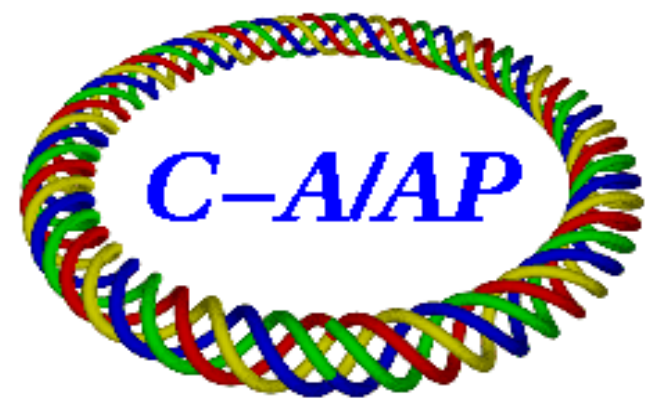

\section{Collider-Accelerator Department Brookhaven National Laboratory Upton, NY 11973}

Notice: This document has been authorized by employees of Brookhaven Science Associates, LLC under Contract No. DE-AC02-98CH10886 with the U.S. Department of Energy. The United States Government retains a nonexclusive, paid-up, irrevocable, world-wide license to publish or reproduce the published form of this document, or allow others to do so, for United States Government purposes. 


\title{
IMPACT ON SPIN TUNE FROM HORIZONTAL ORBITAL ANGLE BETWEEN SNAKES AND ORBITAL ANGLE BETWEEN SPIN ROTATORS*
}

\author{
M. Bai, V. Ptitsyn, T. Roser \\ Collider Accelerator Dept. Upton, NY 11777, U.S.A.
}

\section{Abstract}

To keep the spin tune in the spin depolarizing resonance free region is required for accelerating polarized protons to high energy. In RHIC, two snakes are located at the opposite side of each accelerator. They are configured to yield a spin tune of 1/2. Two pairs of spin rotators are located at either side of two detectors in each ring in RHIC to provide longitudinal polarization for the experiments. Since the spin rotation from vertical to longitudinal is localized between the two rotators, the spin rotators do not change the spin tune. However, due to the imperfection of the orbits around the snakes and rotators, the spin tune can be shifted. This note presents the impact of the horizontal orbital angle between the two snakes on the spin tune, as well as the effect of the vertical orbital angle between two rotators at either side of the collision point on the spin tune.

\section{INTRODUCTION}

The RHIC polarized proton acceleration uses two helical snakes per accelerator to keep the spin tune at $1 / 2$, independent of beam energy and away from all the first order spin resonances at $G \gamma=k$ and $G \gamma=k P+Q_{y}[1,2]$. Here, $G=1.7928474$ is the proton anomalous g-factor. $\gamma$ is the beam Lorentz factor. $\mathrm{k}$ is an integer, $\mathrm{P}$ is the super-periodicity of the lattice. $\mathrm{Q}_{\mathrm{y}}$ is the vertical betatron tune. For RHIC polarized proton operation, $Q_{y}$ is normally set to about 29.72 .

Fig. 1 shows the schematic layout of snakes in one of the RHIC accelerators. Each helical snake rotates the spin vector by $180^{\circ}$ around an axis in the horizontal plane (snake axis). $\phi_{1}$ and $\phi_{2}$ are the angles between two snake axes (red arrows) and the particle's direction (black arrows), respectively. In the absence of machine errors and closed orbit distortions, particles are bent $180^{\circ}$ in between the two snakes and the spinor one turn transfer map is given by

$$
M=e^{-i \frac{\pi}{2}\left(-\sin \phi_{2} \sigma_{1}+\cos \phi_{2} \sigma_{2}\right)} e^{-i \frac{1}{2} G \gamma \pi \sigma_{3}} e^{-i \frac{\pi}{2}\left(\sin \phi_{1} \sigma_{1}+\cos \phi_{1} \sigma_{2}\right)} e^{-i \frac{1}{2} G \gamma \pi \sigma_{3}} .
$$

Here, $\sigma_{i}, i=1,2,3$ are the Pauli matrices in radial, longitudinal and vertical direction, respectively [2]. Eq. 1 shows the stable spin direction is universally along the vertical direction around the accelerator and the spin tune $Q_{s}$ is

$$
\mathrm{Q}_{\mathrm{s}}=\frac{\left|\phi_{1}-\phi_{2}\right|}{\pi}
$$

For RHIC polarized proton operation, $\left|\phi_{1}-\phi_{2}\right|$ is chosen to be $90^{\circ}$ and thus the nominal spin tune in RHIC is $1 / 2$. However, due to the errors, the angle between the two snake axes can deviate from $90^{\circ}$. This then causes a spin tune shift of

$$
\Delta \mathrm{Q}_{\mathrm{s}}=\frac{|\Delta \phi|}{\pi}
$$

Here, $\Delta \phi$ is the amount deviation the angle between the two snake axes away from $90^{\circ}$.

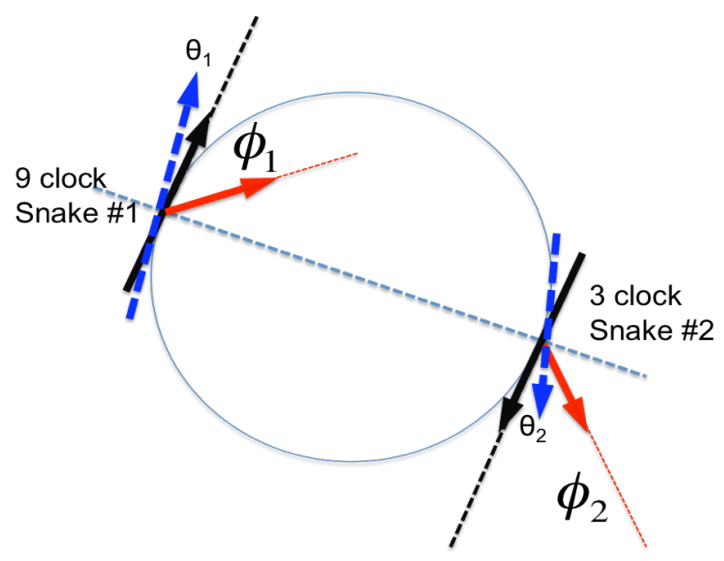

Figure 1: Schematic snake layout in RHIC. The red arrows are the axes of the two snakes. $\phi_{1}$ and $\phi_{2}$ are the angle between the snake axes and the ideal beam directions (black arrows). The dotted blue arrows are the actual beam direction at each snake when horizontal orbital distortion is present. The angle $\theta_{1,2}$ between the blue arrow and black arrow at each snake is the horizontal orbital angle at each snake. between the two snakes. 


\section{SPIN TUNE SHIFT DUE TO ORBITAL ANGLE BETWEEN SNAKES}

Eq. 1 assumes that the amount of spin precessions between the two snakes is exactly $180 \mathrm{G} \gamma$ degrees. In a real machine, this assumption can be broken due to horizontal orbital distortions. This effectively changes the bending angles between the two half rings and causes the spin precesses more in one half of the ring. The pair of dotted blue arrows in Fig. 1 represent the horizontal orbital angles at the two snakes. The angle between the dotted blue arrow and the black arrow $\theta_{1,2}$ is the horizontal orbital angle at snake \#1 and snake \#2, respectively. The horizontal orbital angle between the two snakes $\Delta \theta$ is

$$
\Delta \theta=\theta_{1}-\theta_{2}
$$

i.e. the total bend angle from snake \#1 to snake \#2 clockwise is $\pi-\Delta \theta$ while the total bend angle of the other half of the ring is $\pi+\Delta \theta$. The one turn spin transfer map in the coordinate of the ideal orbit then becomes

$$
M=e^{-i \frac{\pi}{2}\left(-\sin \phi_{2} \sigma_{1}+\cos \phi_{2} \sigma_{2}\right)} e^{-i \frac{1}{2} G \gamma \pi+(1+G \gamma) \Delta \theta \sigma_{3}} e^{-i \frac{\pi}{2}\left(\sin \phi_{1} \sigma_{1}+\cos \phi_{1} \sigma_{2}\right)} e^{-i \frac{1}{2} G \gamma \pi-(1+G \gamma) \Delta \theta \sigma_{3}}
$$

This then yields a spin tune of

$$
Q_{s}=\frac{\left|\phi_{1}-\phi_{2}\right|}{\pi}-(G \gamma+1) \frac{\Delta \theta}{\pi}
$$

with the stable spin direction remaining vertical.

The total spin tune shift due to the snake axes error and horizontal orbital angle between snakes is then

$$
\Delta Q_{s}(\Delta \phi)=Q_{s}-Q_{s, 0}\left(=\frac{1}{2}\right)=\frac{\Delta \phi}{\pi}-(G \gamma+1) \frac{\Delta \theta}{\pi} .
$$

For RHIC, we have beam position monitors at the two quadrupoles Q7 and Q8 on either side of each snake, and beam always travels from quadrupole Q7 to Q8. The horizontal angle between the two snakes in RHIC is defined as the ratio of the beam position difference at Q7 and Q8 and the distance between the two beam position monitors, i.e.

$$
\Delta \theta=\frac{\mathrm{x}_{9, \mathrm{Q} 7}-\mathrm{x}_{9, \mathrm{Q} 8}-\left(\mathrm{x}_{3, \mathrm{Q} 7}-\mathrm{x}_{3, \mathrm{Q} 8}\right)}{\text { distance between } \mathrm{Q} 7 \text { andQ8 }}
$$

where $\mathrm{x}_{\mathrm{i}, \mathrm{Q} 7}$ and $\mathrm{x}_{\mathrm{i}, \mathrm{Q} 8}$ are the horizontal beam positions at quadrupole Q7 an Q8 in sector \#i, $\mathrm{i}=9$ and 3.

Eq. 3 shows the total spin tune shift due to the snake axes not being perfectly perpendicular as well as due to the horizontal orbital angle between the two snakes. For only a fixed snake axes angle error, the spin tune shift is independent of beam energy. However, the contribution from the horizontal orbital angle between snakes grows linearly with beam energy. For a $0.1 \mathrm{mrad}$ orbital angle between the snakes, the amount of spin tune shift is about 0.006 at a beam energy of $100 \mathrm{GeV}$.

For accelerating polarized protons to high energy, it is very critical to set the spin tune as close to $1 / 2$ as possible and keep it constant through the acceleration. Since the spin tune shift comes from both the errors in the snake currents as well as the local closed orbit distortions at the snakes, it is critical to optimize the snake current settings for a given orbital angle between snakes. For the RHIC polarized proton operations, we currently optimize snake settings by measuring beam polarization as a function of snake current at injection with the vertical tune set at 0.745 , close to the snake resonance at $3 / 4$. However, because in RHIC the snake currents are kept constant through the acceleration and the snake axis angle $\phi_{1,2}$ does change during the early part of the acceleration due to the relativistic effect, this snake current scan only allows us to set the spin tune close to $1 / 2$, with an uncertainty of 0.005 , at injection. This change becomes negligible when beam 
gets very relativistic, and the contribution from the horizontal orbital angle, on the other hand, is linearly proportional to the beam energy. Hence, the spin tune can get away from $1 / 2$ as beam is accelerated to higher energy and it is important to differentiate the amount of spin tune shift due to the snake errors and due to the orbital angle between the two snakes at the injection. This can be achieved by measuring the spin tune change as a function of the snake orbital angle at two different energies for instance, $100 \mathrm{GeV}$ and $250 \mathrm{GeV}$, where the snake axis becomes independent of the beam energy for a given snake current. In addition, it is also critical to have precise control of the orbital angle between the snakes.

According to the RHIC spin tracking studies, the tolerance on the spin tune shift is around 0.005. This means we have to control the orbital angle between snakes through the acceleration to $0.1 \mathrm{mrad}$ or better. So far, the achieved horizontal orbital angle between the two snakes is about 0.3-0.4 mrad. Fig. 2 shows the measured horizontal orbital angle between the two snakes in Blue ring at the four strong intrinsic spin resonances during the acceleration up to $100 \mathrm{GeV}$ for the RHIC polarized proton run in 2005, 2006 and 2008. The orbital angle was calculated from the beam position monitor data at Q7 and Q8 during the past RHIC polarized proton runs. The large scattering of the orbital angle in 2005 is very likely due to the beam position monitor problems. No orbital angle data for the Yellow ring snakes during the RHIC polarized proton run in 2008 was available due to a broken feed-through of the beam position monitor at quadrupole Q8 at 9'clock in Yellow ring.

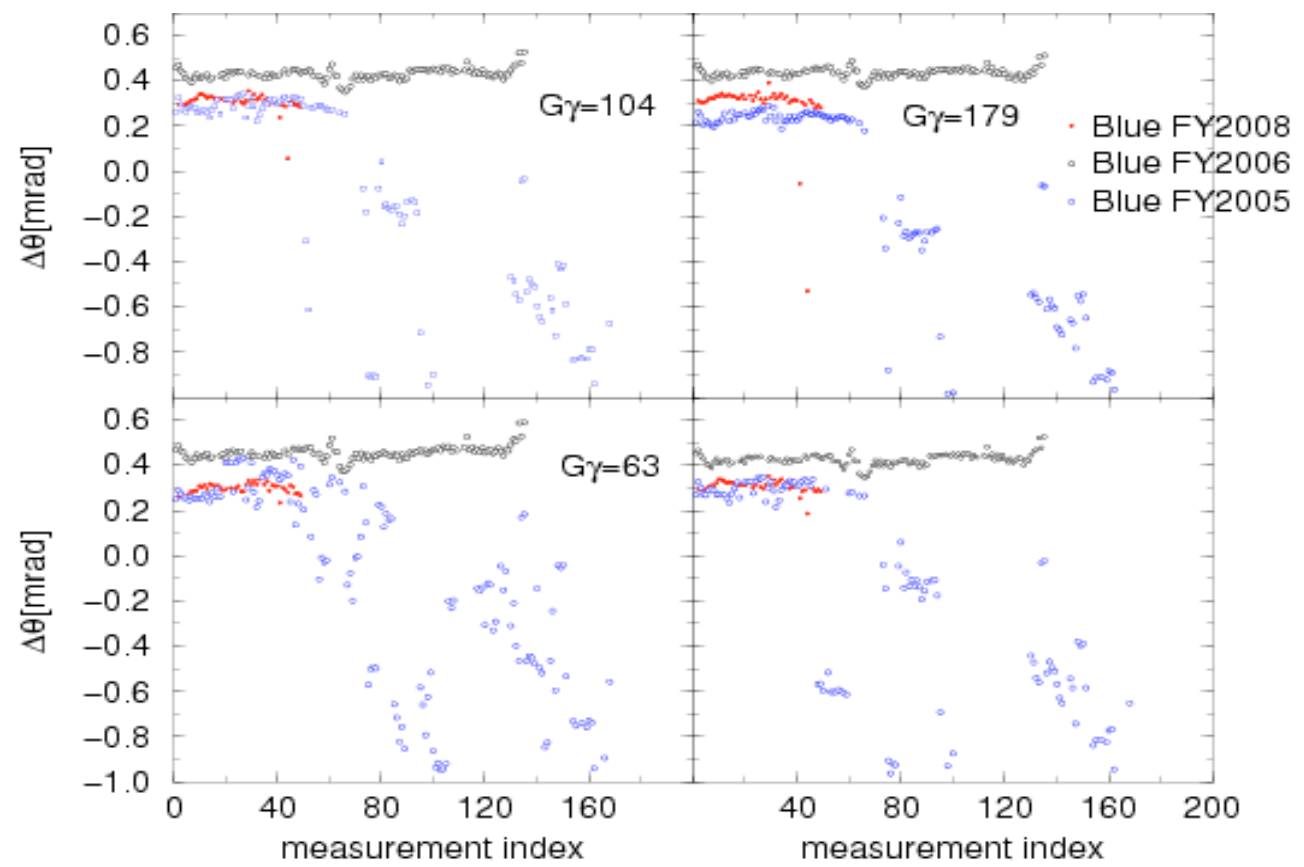

Figure 2: measured horizontal orbital angles between the two snakes in the Blue ring at the four strong intrinsic depolarizing resonances . This is based on the RHIC beam position monitors at either side of each snake.

During the summer shut down after the RHIC polarized proton operation in 2008, a survey of the magnets around the snake areas and spin rotator areas was conducted. Fig. 3 shows the calculated horizontal orbital angle between the two snakes calculated from the latest survey data [3]. Based on these data, the horizontal angle between the two snakes in the Blue ring and Yellow ring is $-0.03 \mathrm{mrad}$ and $-0.11 \mathrm{mrad}$, respectively. The larger angle due to mis-alignment in the Yellow ring could have lead to the lower polarization transmission efficiency and re-alignment of the quadrupoles in the snake areas are in progress. 


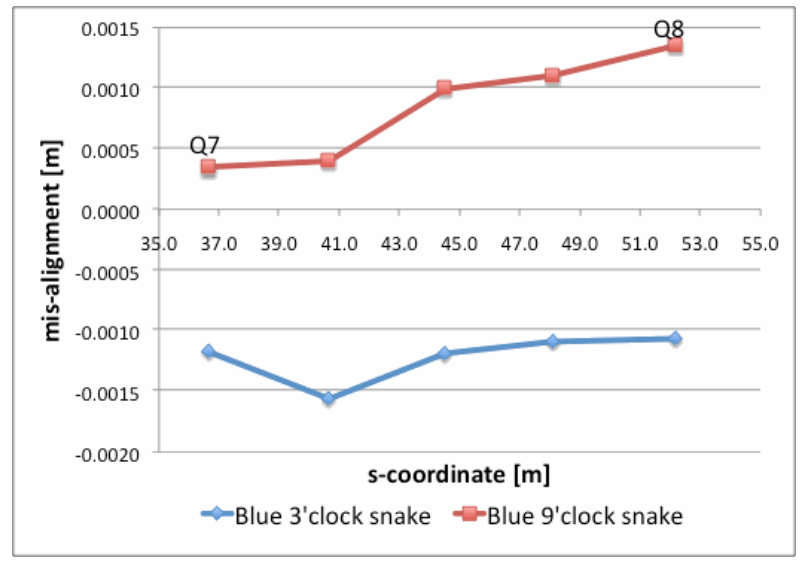

Figure 3a: Horizontal orbital angle between the two snakes in the Blue ring based on the latest RHIC survey data.

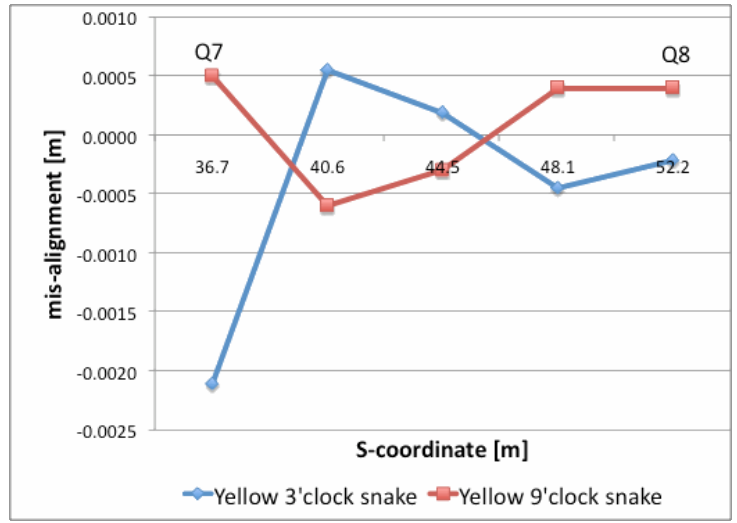

Figure 3b: Horizontal orbital angle between the two snakes in the Yellow ring based on the latest RHIC survey data

\section{SPIN TUNE SHIFT DUE TO THE ORBITAL ANGLE BETWEEN ROTATORS}

In order to provide longitudinal spin direction, the two physics detectors STAR and PHENIX at RHIC are equipped with two pairs of spin rotators [4]. Unlike snakes, each spin rotator rotates the spin vector by a fraction of $180^{\circ}$. The spin rotators are arranged in pairs to keep the stable spin direction vertical for the rest of the ring. Ideally, there are no net bends, i.e. additional spin rotations, between the two spin rotators. In this case, one spin rotator on one side of the detector first brings the spin vector from vertical to the horizontal plane. Then the spin rotator on the other side brings the spin vector back to vertical. Since this arrangement localize the non-vertical spin direction between the two rotators, the spin tune remains $1 / 2$ and the stable spin direction also remains vertical for the rest of the ring.

In RHIC, there are two dipole magnets DX and D0 located between each spin rotator and the interaction point [4]. Since the net bending of DX and D0 is not zero, they precess the spin rotator by an angle $\phi=\mathrm{G} \gamma *\left(\theta_{\mathrm{DX}}+\theta_{\mathrm{D} 0}\right)$. Here, $\theta_{\mathrm{DX}}$ and $\theta_{\mathrm{D} 0}$ are the bend angles of DX and D0 magnets, respectively. For the head-on collisions, the total bend of two pairs of DX and D0 on either side of the interaction point is zero, there is no net spin precession from the two pairs of DX and D0 magnets. Since the stable spin direction between the spin rotators is in the horizontal plane, a vertical orbital deflection between the spin rotators (a vertical orbital angle between the spin rotators) can lead to a spin tune change. In RHIC, it is a drift space between the two DX magnets, the only possible source of orbital deflection comes from the region between spin rotator and DX\&D0 pair where triplet is located to provide final beta squeeze at the interaction point.

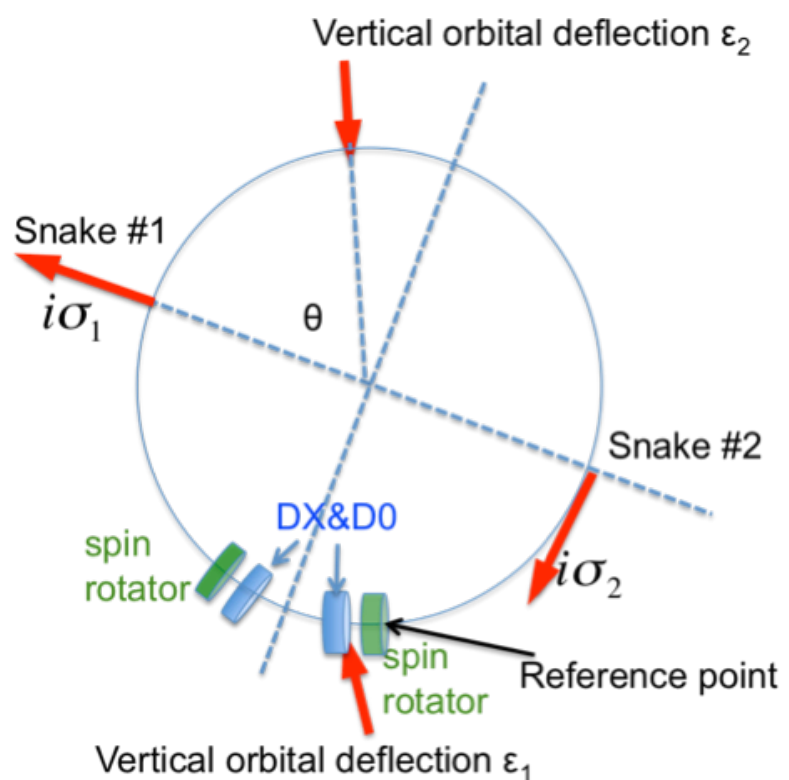

Figure 4: Schematic layout of RHIC with one pair of spin rotators. To simplify the calculation, the bend between each snake to the spin rotator pair is $\pi / 2$. The one turn transfer matrix is calculated for the reference point at the entrance of the spin rotator on the right side of the interaction point. Here, beam travels clockwise. A vertical orbital deflection $\varepsilon_{1}$ is located in between the spin rotator and the DX\&D0 magnet. A vertical orbital deflection $\varepsilon_{2}$ outside the spin rotator pair located at a location $\theta$ away from the first snake $\left(i \sigma_{1}\right)$ is required to keep the vertical orbit closed. 
Without loosing generality, we first evaluate the simple model in Fig. 4 with one pair of spin rotators located in between the two snakes. To simplify the calculation, either snake is located to be at a bend of 90 degrees away from the spin rotators. To calculate the spinor one turn transfer matrix, we assume that the reference point is at the entrance of the spin rotator at the right side of the interaction point and the particle travels clockwise. A vertical orbital deflection $\varepsilon_{1}$ is located between the spin rotator and the adjacent DX\&D0 magnet. Another vertical orbital deflection located outside the spin rotator pair is required to keep the vertical orbit closed.

To provide a stable spin direction in the horizontal plane, each spin rotator is configured to rotate the spin vector by 90 degrees around an axis $\vec{n}=n_{1} \hat{x}+n_{2} \hat{z}$. Here, $\hat{x}$ and $\hat{z}$ are the radial and longitudinal unit vector respectively. The spinor one turn transfer matrix is then given by

$$
M=e^{-\frac{i}{2} G \gamma \frac{\pi}{2} \sigma_{3}}\left(i \sigma_{2}\right) e^{-\frac{i}{2} G \gamma(\pi-\theta) \sigma_{3}} e^{-\frac{i}{2}(1+G \gamma) \varepsilon_{2} \sigma_{1}} e^{-\frac{i}{2} G \gamma \theta \sigma_{3}}\left(i \sigma_{1}\right) e^{-\frac{i}{2} G \gamma \frac{\pi}{2} \sigma_{3}} e^{\frac{i}{2} \frac{\pi}{2}\left(n_{1} \sigma_{1}+n_{2} \sigma_{2}\right)} e^{-\frac{i}{2}(1+G \gamma) \varepsilon_{1} \sigma_{1}} e^{-\frac{i}{2} \frac{\pi}{2}\left(n_{1} \sigma_{1}+n_{2} \sigma_{2}\right)}
$$

where

$$
\left(\begin{array}{l}
n_{1} \\
n_{2}
\end{array}\right)=\left(\begin{array}{c}
-\cos (\phi+\psi) \\
\sin (\phi+\psi)
\end{array}\right)
$$

Here, $\psi$ is the angle of between the stable spin direction at interaction point and the beam direction. $\varepsilon_{1}$ is the vertical orbital deflection the spin vector encounter between the D0 magnet and spin rotator. To simplify the problem, we assume a single orbital deflection is located outside the spin rotator pair at a bend angle $\theta$ away from snake $\left(i \sigma_{1}\right)$. The deflection $\varepsilon_{2}$ is selected to keep the orbit closed together with epsilon one. Eq. 9 then gives spin tune change as

$$
\sin \left(\Delta Q_{s} \pi\right)=-\left[n_{2} \cos \frac{(1+G \gamma) \varepsilon_{2}}{2} \sin \frac{(1+G \gamma) \varepsilon_{1}}{2}-n_{1} n_{2} \sin \left(\frac{(1+G \gamma) \varepsilon_{1}}{2}\right) \sin \left(\frac{(1+G \gamma) \varepsilon_{2}}{2}\right) \cos \left(G \gamma\left(\frac{\pi}{2}-\theta\right)\right)-n_{1}^{2} \sin \left(\frac{(1+G \gamma) \varepsilon_{1}}{2}\right) \sin \left(\frac{(1+G \gamma) \varepsilon_{2}}{2}\right) \sin \left(G \gamma\left(\frac{\pi}{2}-\theta\right)\right)\right] .
$$

In general for a given energy, since the last two terms in Eq. (11) are quadratic to the orbital deflection, the maximum tune change is on the order of $10^{-4}$. Their contribution is negligible compare to the linear term. Hence, the spin tune change is independent of the location of orbital deflection $\varepsilon_{2}$ unless $n_{2}=0$.

For RHIC, the nominal total bend angle of DX\&D0 is about $3.7 \mathrm{mrad}$. This leads to a total of spin rotation of 40.6 degrees at $100 \mathrm{GeV}$ and 100.4 degrees at $250 \mathrm{GeV}$. So, for the case of longitudinal stable spin direction at interaction point, i.e. $\psi=0$, the spin tune change dominated by the first term in Eq. (11) which is about linear with beam energy for a fixed orbital deflection. For $\varepsilon_{1}=-\varepsilon_{2}=0.1 \mathrm{mrad}$ orbital deflection, spin tune change is 0.002 at $100 \mathrm{GeV}$ and 0.0075 at $250 \mathrm{GeV}$.

On the other hand, for the case of radial stable spin direction at interaction point, ie. $\psi=90^{\circ}$, the sum of $\phi+\psi$ in Eq. 10 and Eq. 11 goes from 130.6 degrees to 190.4 degrees for beam energy ranging from $100 \mathrm{GeV}$ to $250 \mathrm{GeV}$. For $\varepsilon_{1}=-\varepsilon_{2}=$ $0.1 \mathrm{mrad}$ orbital deflection, the spin tune change is about 0.0023 at $100 \mathrm{GeV}$ and -0.0014 at $250 \mathrm{GeV}$.

Compare with the real machine, Eq. 11 is an approximation for estimating the spin tune change due to the vertical orbital angle between spin rotators. In order to get more accurate calculation, one needs to include the following

1. Include both spin rotator pairs at interaction point for PHENIX as well as for STAR

2. Solenoid fields between the spin rotators

All these effects will be investigated and reported in the future tech note. Nevertheless Eq. 11 provide a qualitative estimate on the sensitivity of the spin tune change on the vertical orbital angle between spin rotators. Overall, Eq. 11 shows that for a fixed energy, the spin tune change is less sensitive to the vertical orbital angle between spin rotators than to the horizontal orbital angle between snakes. It also shows that similar to the orbital angle between snakes. This also suggests that one would prefer to only turn on the spin rotators at store energy where the nearby spin depolarizing resonances are weak.

The above discussions assume zero horizontal orbital angle between each spin rotator pairs. Similar to the horizontal angles between snakes, the horizontal angles between the entrance and exit of each spin rotator pair also contribute to 
spin tune change. To evaluate the contribution of horizontal orbital angle to the spin tune change, let's evaluate a simple case of one pair of snakes with two pairs of spin rotators. Here, we assume the two snakes $\left(i \sigma_{1}\right)$ and $\left(i \sigma_{2}\right)$ are on the opposite side of the ring. Starting from the snake $\left(i \sigma_{1}\right)$ going clockwise, one pair of the spin rotators is located at $\phi_{21}$ away from the snake with horizontal orbital angle $\theta_{2}$ and $\theta_{3}$ at the entrance and exit of the first spin rotator pair. The second spin rotator pair is located at $\phi_{43}$ away from the first spin rotator pair with horizontal orbital angle $\theta_{4}$ and $\theta_{5}$ at the entrance and exit of the second spin rotator pair. The horizontal orbital angle at snake $\left(i \sigma_{1}\right)$ and $\left(i \sigma_{2}\right)$ is $\theta_{1}$ and $\theta_{6}$, respectively. Without loosing generality, we also assume each spin rotator rotates the spin vector 90 degrees around an axis in radial direction. The one turn spinor transfer map is then given by

$$
\begin{aligned}
& M=\left(i \sigma_{1}\right) e^{-\frac{i}{2}\left[G \gamma \pi+(1+G \gamma)\left(\theta_{6}-\theta_{1}\right)\right] \sigma_{3}}\left(i \sigma_{2}\right) e^{\frac{i}{2}(1+G \gamma) \theta_{6} \sigma_{3}} e^{-\frac{i}{2} G \gamma \phi_{65} \sigma_{3}} e^{-\frac{i}{2}(1+G \gamma) \theta_{5} \sigma_{3}} e^{\frac{i}{2} \frac{\pi}{2} \sigma_{1}} e^{\frac{i}{2}(1+G \gamma)\left(\theta_{5}-\theta_{4}\right) \sigma_{3}} e^{-\frac{i}{2} \frac{\pi}{2} \sigma_{1}} e^{\frac{i}{2}(1+G \gamma) \theta_{4} \sigma_{3}} \\
& e^{-\frac{i}{2} G \gamma \phi_{43} \sigma_{3}} e^{-\frac{i}{2}(1+G \gamma) \theta_{3} \sigma_{3}} e^{\frac{i}{2} \frac{\pi}{2} \sigma_{1}} e^{\frac{i}{2}(1+G \gamma)\left(\theta_{3}-\theta_{2}\right) \sigma_{3}} e^{-\frac{i}{2} \frac{\pi}{2} \sigma_{1}} e^{\frac{i}{2}(1+G \gamma) \theta_{2} \sigma_{3}} e^{-\frac{i}{2} G \gamma \phi_{21} \sigma_{3}} e^{-\frac{i}{2}(1+G \gamma) \theta_{1} \sigma_{3}}
\end{aligned}
$$

where $\phi_{65}=\pi-\phi_{21}-\phi_{43}$. After simplification of Eq. 12, one can then calculate the spin tune change is

$$
\sin \left(\Delta Q_{s} \pi\right)=-\cos \left[\frac{1+G \gamma}{2} \theta_{32}\right] \cos \left[\frac{1+G \gamma}{2} \theta_{54}\right] \sin \left[(1+G \gamma) \theta_{61}-\frac{1+G \gamma}{2}\left(\theta_{32}+\theta_{54}\right)\right]
$$

where $\theta_{j i}=\theta_{j}-\theta_{i}$ and $\mathrm{i}, \mathrm{j}$ are integers from 1 to 6 . For very small $\frac{1+G \gamma}{2} \theta_{32}$ and $\frac{1+G \gamma}{2} \theta_{54}$, the spin tune change is

$$
\Delta Q_{s} \approx \frac{1+G \gamma}{\pi}\left(\theta_{61}-\frac{\theta_{32}}{2}-\frac{\theta_{54}}{2}\right)
$$

Compare with Eq. 6, the maximum spin tune change due to the horizontal orbital angle between the exit and entrance of each spin rotator pair has similar behavior with the spin tune change due to the horizontal orbital angle between snakes.

\section{CONCLUSIONS}

To have precise spin tune control is very important for accelerating polarized protons to high energy. For RHIC, it is shown that spin tune shift can be caused by not only the imperfection of snake configurations but also the local orbital distortion at snake and spin rotators. These two effects have different energy dependence on the beam energy. For fixed snake current, the spin tune shift due to the snake axes not being perfectly perpendicular remains constant when particle gets to high energy. The effect on spin tune due to the local horizontal orbital angle between two snakes and vertical orbital angle between the two spin rotators on the other hand grow with beam energy. Hence, it is very critical to have precise control of the local orbit around snakes to avoid depolarization.

In RHIC, two pairs of spin rotators are also employed at either side of the two physics detectors in each ring to provide polarized proton collisions with longitudinal or radial spin direction. Since the stable spin direction between the two rotators are in the horizontal plane, the vertical orbital angle between the two spin rotators can also produce spin tune shift. The evaluation of spin tune change due to the vertical orbital angle between spin rotators of a simplified RHIC like model shows that the spin tune change due to the orbital angle between spin rotators also grows with beam energy. This means accelerating polarized proton with fully powered spin rotator requires much tighter constraint on the local orbit control around spin rotators. Hence, it is preferred for the polarized proton operation to keep the spin rotators off until when beam reaches the store energy where the intrinsic resonances are weak.

\section{ACKNOWLEGEMENT}

The authors would like to thank Todd Satogata for helping to get the data of the beam position monitors around snakes. The authors also would like to thank Frank Karl for providing the survey data of the snake and spin rotator areas.

\section{REFERENCES}

[1] RHIC Polarized Proton Design Manual,

[2] Spin Dynamics and Snakes in Synchrotrons, S. Y. Lee, World Scientific Publishing Company

[3] Frank Karl's presentation 
